\title{
Intelligent Control of an Uncertain Distillation Column Using a Multivariable Filter Decoupling-based PID Like Fuzzy Controller
}

\author{
Niloofar Mirzavand ${ }^{1}$, Farzin Piltan ${ }^{2}$ and Jong-Myon Kim ${ }^{2, *}$ \\ ${ }^{1}$ Control and Robotic Lab, IRAN SSP Research and Development Center, \\ Shiraz, Iran \\ ${ }^{2}$ School of Electrical and Electronic Engineering, University of Ulsan, \\ Ulsan 680-749, South Korea \\ Piltan_f@iranssp.org, jongmyon.kim@gmail.com
}

\begin{abstract}
The main objective of this paper is the design of a robust and stable multivariable decoupling based Proportional-Integral-Derivative (PID) like fuzzy scheduling technique to control multi-input, multi-output (MIMO) nonlinear uncertain dynamical distillation column. A PID like fuzzy scheduling controller was used in this paper to control the distillation column in the presence of uncertainty and disturbance. To address the challenges of robustness, stability, and error convergence under uncertain conditions, the proposed multivariable decupling method was applied to the PID like fuzzy scheduling method. This theory was applied to a P-canonical form and $V$-canonical form of distillation column modeling to verify the power of the control, stability and robustness proposed method.
\end{abstract}

Keywords: Multivariable decoupling method, distillation column, PID like fuzzy scheduling method, P-canonical system modeling, V-canonical system modeling, stability, robustness

\section{Introduction}

The principal goal of this research was to design a robust nonlinear multivariable decoupling technique to improve the stability, robustness and performance of the PID fuzzy scheduling controller for distillation column. The architecture of the distillation columns have complexities in that these systems behave nonlinearly with uncertainties in the parameters, disturbance and couples. Based on the distillation column's organizational properties, the design of a high-performance controller is noteworthy for isolation of fluids $[1,2]$. Conventional control algorithm, intelligent control technique and hybrid method are the main methods to control of distillation columns. Linear based control techniques and nonlinear model-reference control methods are the main algorithms to design conventional controller for linear and nonlinear systems.

Linear control algorithm is used in numerous industries, but it cannot guarantee three main factors to control applications, namely, stability, robustness and reliability. The main linear control algorithm adventures a proportional derivative (PD) control theory, proportional integral (PI) control algorithm, and proportional integral derivative (PID) control technique. The second type of conventional control algorithms exploits a nonlinear model-reference control technique. The main nonlinear model-reference controllers are the sliding mode controller, computed torque controller, back-stepping controller and, Lyapunov-based controller. Fuzzy based algorithm, neural network theory, neuro-fuzzy method and genetic algorithm method are the foremost techniques to design

Received (October 29, 2017), Review Result (December 7, 2017), Accepted (January 9, 2018)

* Corresponding Author 
artificial intelligent controller for linear and nonlinear systems. Selecting a suitable control algorithm is a major target for many researchers in this field [3-7].

The coupling effect is the significant challenge to select the control technique for the distillation column. If linear techniques are contenders, there are two challenges to overcome with regard to the coupling effect: limitation the time of the system performance, and reduce the controller's sensitivity. To solve the challenge of uncertainty, we used intelligent techniques in this paper. An adaptive output fuzzy feedback control for completely uncertain nonlinear switched system, has been developed by Yongming Li et.al., [8]. The fuzzy logic in this paper was used for modeling uncertain system and adaptive fuzzy back-stepping method was used to control. The challenges of system identification and control of single input-single output, nonlinear uncertain, unmodeled dynamic, dynamic disturbance, and unknown time delays system was presented by Shen et al., Yin. [9]. In this paper authors were used the Lyapunov function to compensate the uncertain parameters. The application of fuzzy logic in back-stepping controller to improve the system convergence in uncertain nonstrict-feedback systems has been presented by Qi Zhou et. al., [10]. To speed control of single-axis rotation system dual close loop controller was presented by Jun Shen et.al. [11]. In this research they had been designed PID to position control and tracking differentiator based fuzzy PID used for speed control. In this paper they have been improved the performance of fuzzy logic controller by adaptive algorithm. According to [8-11] the fuzzy logic theory was improved the nonlinear techniques to control of nonlinear and uncertain systems. Apart from several advantages, nonlinear based control method has challenges associated with industrial system applications. Various researcher were used industrial techniques to control of distillation column [12-14]. Stefano Ciannella [12] presented industrial hotvapor bypass method to pressure control in distillation columns to separation of water and ethanol. The asymmetrical temperature controller for BTX dividing-wall distillation column was given by Yang Yuan et al. [13]. The plant-wide control for coupled distillation columns with partial condensers has been presented by Ebrahimzadeh and Baxter [14].

A PID scheduling fuzzy controller is proposed to solve the robustness challenge in an industrial PID controller. Apart from several advantages in particular conditions, this method contains the coupling challenge in uncertainty. A proposed multivariable decoupling method is used to modify the PID scheduling fuzzy controller by applying a multivariable decoupling theory to reduce the system nonlinearity and design MIMO controller based on SISO. The rest of this paper is organized as follows. The second part outlines the theory of distillation columns. In the third part, the problem statements and the proposed multivariable decoupling-based fuzzy PID scheduling controller are presented. In the fourth part, we analyzed the proposed method. In the final section, we provide conclusions.

\section{Problem Statement}

The distillation column is highly nonlinear and possesses a multi-input multi-output (MIMO), coupling effect, and uncertainty of the dynamic parameters. This system is used in oil and gas industries and is work in unknown and unstructured conditions. Linear modelling is no certify that the resulting controller is closely matched to the real system. To solve this challenge multivariable modelling is recommended because the resulting of control strategies perform is depended on system modelling accuracy. The V-canonical modeling of distillation column is considered in the Figurel shown below. The $2 \times 2 \mathrm{~V}$ canonical input/output of distillation column is considered in the equation shown below.

$$
\left\{\begin{array}{l}
Y_{1}=\left[Y_{2} W_{12}^{V}+U_{1}\right] W_{11}^{V} \\
Y_{2}=\left[Y_{1} W_{21}^{V}+U_{2}\right] W_{22}^{V}
\end{array}\right.
$$


where $Y_{i}, U_{i}$ are output and input, respectively. According to (1), the following relationship can be obtained.

$$
Y=\left[I-W_{m}^{V} W_{i}^{V}\right]^{-1} W_{m}^{V} U
$$

where $W_{m}^{V}=\left(\begin{array}{cc}W_{11}^{V} & 0 \\ 0 & W_{22}^{V}\end{array}\right)$ and $W_{i}^{V}=\left(\begin{array}{cc}0 & W_{12}^{V} \\ W_{21}^{V} & 0\end{array}\right)$. Based on (2), in the first step to address the challenge of the coupling effect in the distillation column, a multivariable decoupling is presented in this paper. In the second phase, fuzzy PID scheduling controller is applied to the multivariable decoupling method to address the uncertainty challenge.

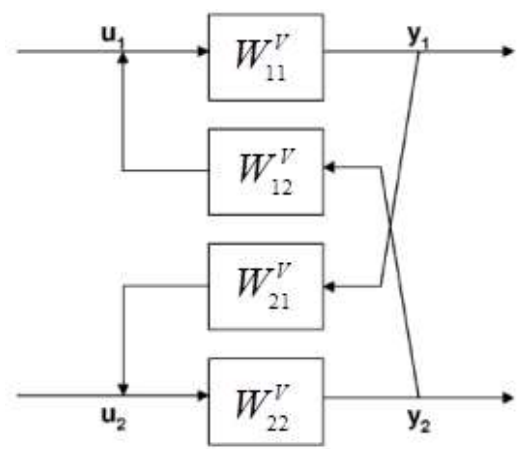

Figure 1. V-Canonical Form of Modelling for Distillation Column

\section{Proposed Methodology}

Reducing the coupling effect is the first objective in this research, and the multivariable decoupling methodology is the best candidate. This algorithm has two main parts: 1) a Pcanonical modelling of distillation column and 2) a series multivariable decoupled. The first part is used to improve the system modeling in distillation column. The P-canonical modeling of distillation column is considered in the Figure 2 shown below.

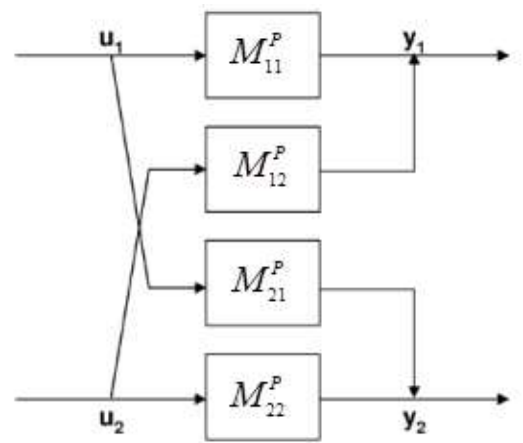

Figure 2. P-canonical Form of Modelling for Distillation Column

The $2 \times 2$, P-canonical input/output of distillation column is considered in the equation shown below. 


$$
\left\{\begin{array}{l}
Y_{1}=M_{11}^{P} U_{1}+M_{12}^{P} U_{2} \\
Y_{2}=M_{21}^{P} U_{1}+M_{22}^{P} U_{2}
\end{array}\right.
$$

where $Y_{i}, U_{i}$ are output and input, respectively. The P-canonical modeling is simpler than $\mathrm{V}$-canonical type of modeling.

Design a decoupling control scheme is the main prevalent technique to control of coupling effect MIMO system. The main role of controller is to maintain bottom and top process purity despite variations. To eliminate the completely effect of the loop interactions, compensation decouple technique is recommended. The main contribution of system decoupling is decomposed a multivariable system into the independent single loop system. Figure 3 shows the P-canonical decoupling method for distillation column.

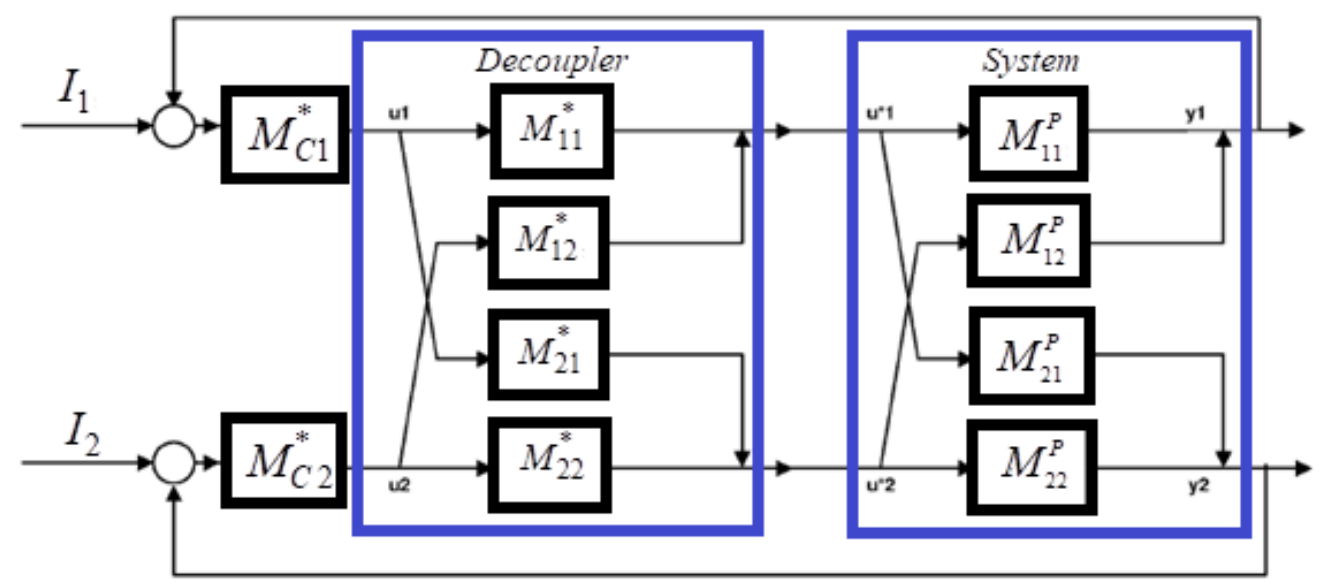

Figure 3. P-canonical Decoupling Method for Distillation Column

In distillation column the system inputs are heat $(E)$ and the rate of reflux ratio $(\varepsilon)$.

$$
\varepsilon=\frac{L}{V_{k}}
$$

Where $L, V_{k}$ are reflux and vapor flow, respectively. Based on Figure 3 if distillation column has disturbance the system can be formulated as follows.

$$
\left\{\begin{array}{l}
Y_{1}=M_{11}^{P} U_{1}+M_{12}^{P} U_{2}+d_{1} \\
Y_{2}=M_{21}^{P} U_{1}+M_{22}^{P} U_{2}+d_{2}
\end{array}\right.
$$

According to (5), the system formulation is proved as follows:

$$
\begin{aligned}
& {\left[\begin{array}{l}
Y_{1} \\
Y_{2}
\end{array}\right]=\left[\begin{array}{ll}
M_{11} & M_{12} \\
M_{21} & M_{22}
\end{array}\right]\left[\begin{array}{l}
\Delta \varepsilon \\
\Delta E
\end{array}\right]} \\
& {\left[\begin{array}{l}
Y_{1} \\
Y_{2}
\end{array}\right]=\left[\begin{array}{lc}
\frac{-1248}{1+6.9 S} & \frac{140}{(1+1.26 S)(1+1.58 S)} \\
\frac{-1590}{1+2.9 S} & \frac{344}{1+1.69 S}
\end{array}\right]\left[\begin{array}{l}
\Delta \varepsilon \\
\Delta E
\end{array}\right]}
\end{aligned}
$$

where $Y_{1}, Y_{2}$ are the difference of two product temperature, respectively. According to (6), control of product temperature instead of concentrations is the main proposed. To 
design decoupling block for steady state condition the frequency in (5) go toward the zero. The decoupling formulation of system is proven as follows:

$$
\left\{\begin{array}{l}
M_{12}(0)+E_{12} M_{22}(0)=0 \\
M_{21}(0)+E_{21} M_{11}(0)=0 \\
E_{12}=\frac{G_{12}(0)}{G_{22}(0)}=5.63 \\
E_{21}=-\frac{G_{21}(0)}{G_{11}(0)}=0.09
\end{array}\right.
$$

The transfer function of the resulting main paths of the process is obtained as follows:

$$
\left\{\begin{array}{c}
M_{1_{F}}(s)=M_{11}^{P}(s)+M_{21}^{*} \times M_{12}^{P}(s)=-800 \frac{1+0.82 S+3.3 S^{2}}{1+8.9 S+19 S^{2}+11.1 S^{3}} \\
M_{2_{F}}(s)=M_{22}^{P}(s)+M_{12}^{*} \times M_{21}^{P}(s)=210 \frac{1+3.9 S}{1+5.4 S+4.9 S^{2}}
\end{array}\right.
$$

Figure 4 illustrates the step response in the main branch $\left(M_{1_{F}}(s)\right)$ in two different conditions: 1) after multivariable decoupling method, and 2) before multivariable decoupling method.

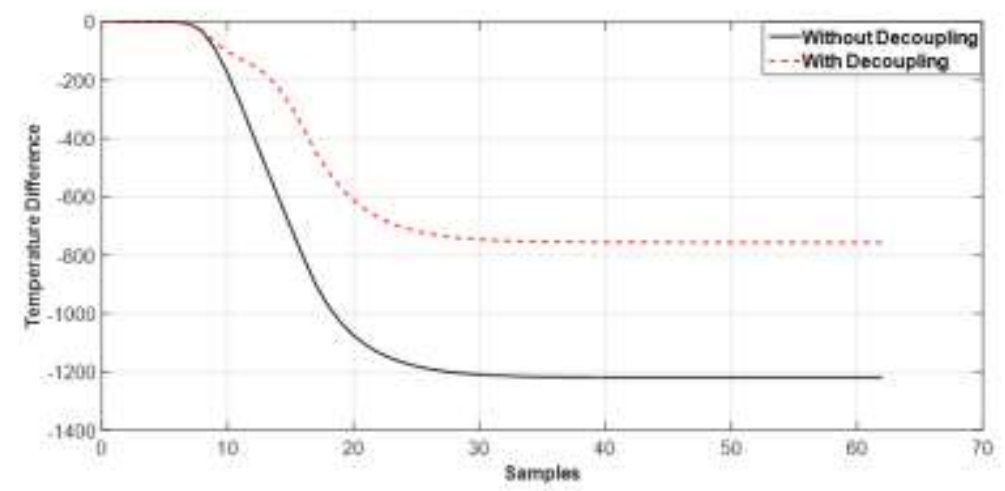

\section{Figure 4. Step Response of the branch 1, Without Decoupling Method and with Decoupling Method}

The second main branch $\left(M_{2_{F}}(s)\right)$ in two different condition is illustrated in Figure 5. Based on Figures 4 and 5, decoupling method reduces the variation of temperature to improve the stability of distillation column. Figures 6 and 7 illustrate the effect of first input in second output and the effect of second input in the first output. According to these two graphs the decoupling methods reduce the effect of coupling in distillation column. Based on Figures 4 to 7, multivariable decoupling method was reduced the effect of nonlinearity and decoupling in distillation column. Due to the multivariable decoupling is applied to the distillation column there will be dynamic cross-over (coupling effect) between the two parts of circuit. To eliminate the dynamic cross-over in distillation column, active low pass filter is recommended. The frequency response for main branch (1) and main branch (2) are shown in Figure 8 and Figure 9. 


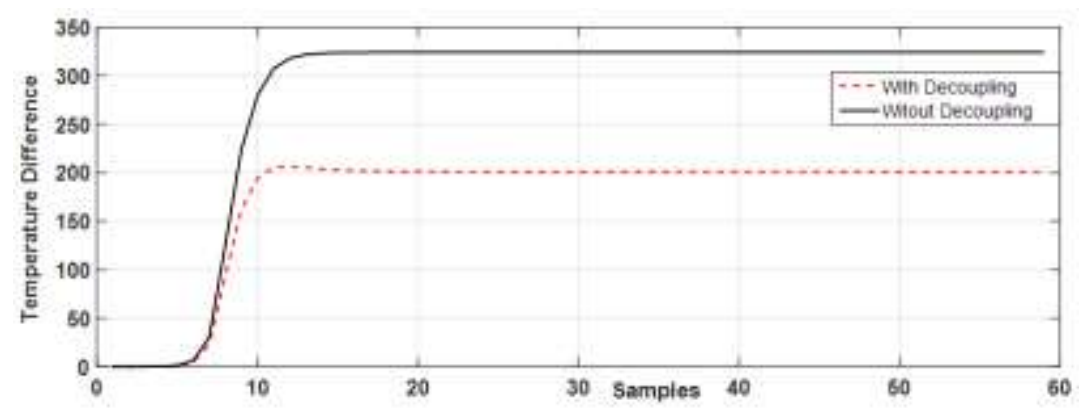

Figure 5. Step Response of the Branch 2, without Decoupling Method and with Decoupling Method

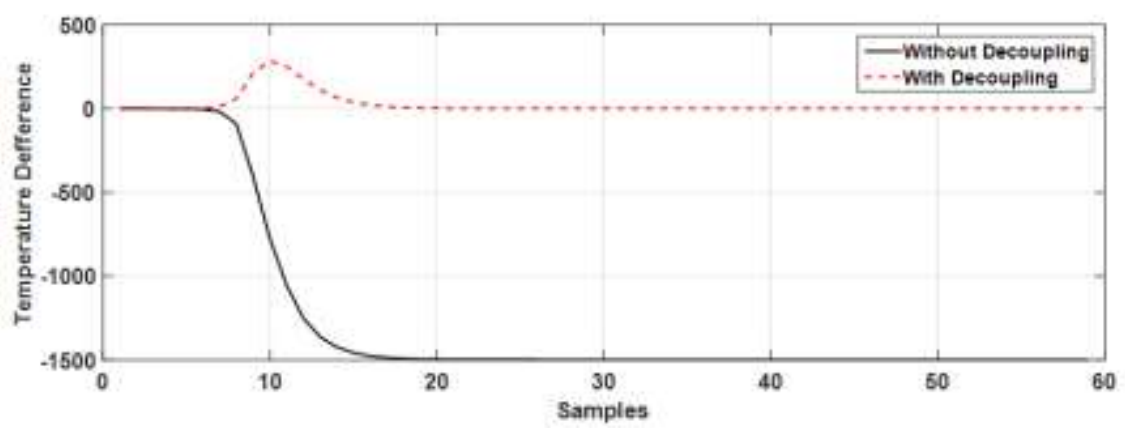

Figure 6. Step Response of the branch 1 to 2, without Decoupling Method and with Decoupling Method

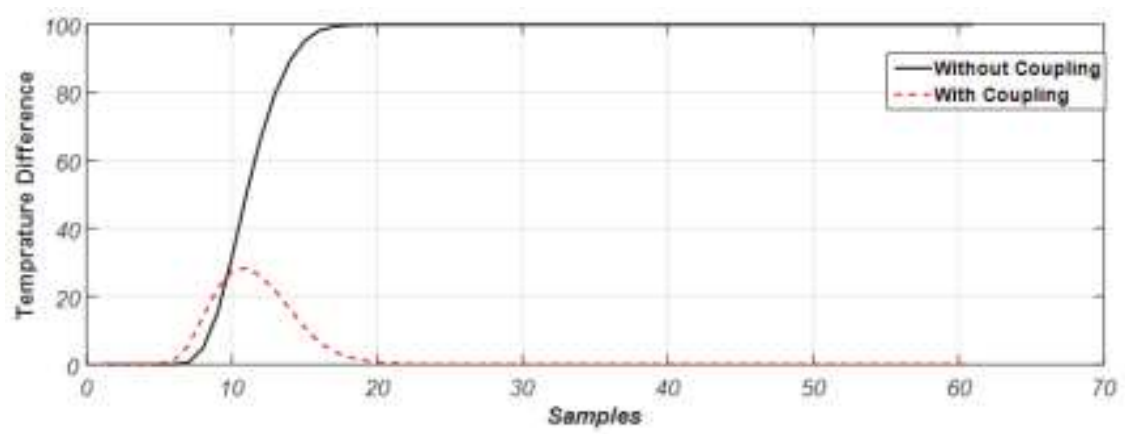

Figure 7. Step Response of the Branch 2 to 1, without Decoupling Method and with Decoupling Method

Based on Figures 8 and 9, correction filter makes the distillation column more stable in a range of frequencies. Nevertheless, multivariable filter decoupling method solves the dynamic cross-over effect, but cannot guarantee steady state error convergence. There are two major factors which must be considered to address this challenge. The first one is dependence on the design PID controller and the second is design fuzzy scheduling theory. To solve the first issue, a modified PID controller is presented. In this case, two PID controllers for the main system decoupling path are designed. This controller has two main parts:1) the cascade of I-controller and 2) the correcting lead PD filter. Based on Figure $3, M_{C_{1}}^{*}, M_{C_{2}}^{*}$ are controllers that designed based on the fuzzy PID scheduling. The PD-based filter controllers are defined by ( 9 and 10).

$$
U_{P D_{1}}=K_{1} \frac{\left(S^{2} T_{1}^{2}+S T_{2}+1\right)\left(1+S T_{3}\right)}{\left(S^{3} T_{4}^{3}+S^{2} T_{5}^{2}+S T_{6}+1\right)}
$$




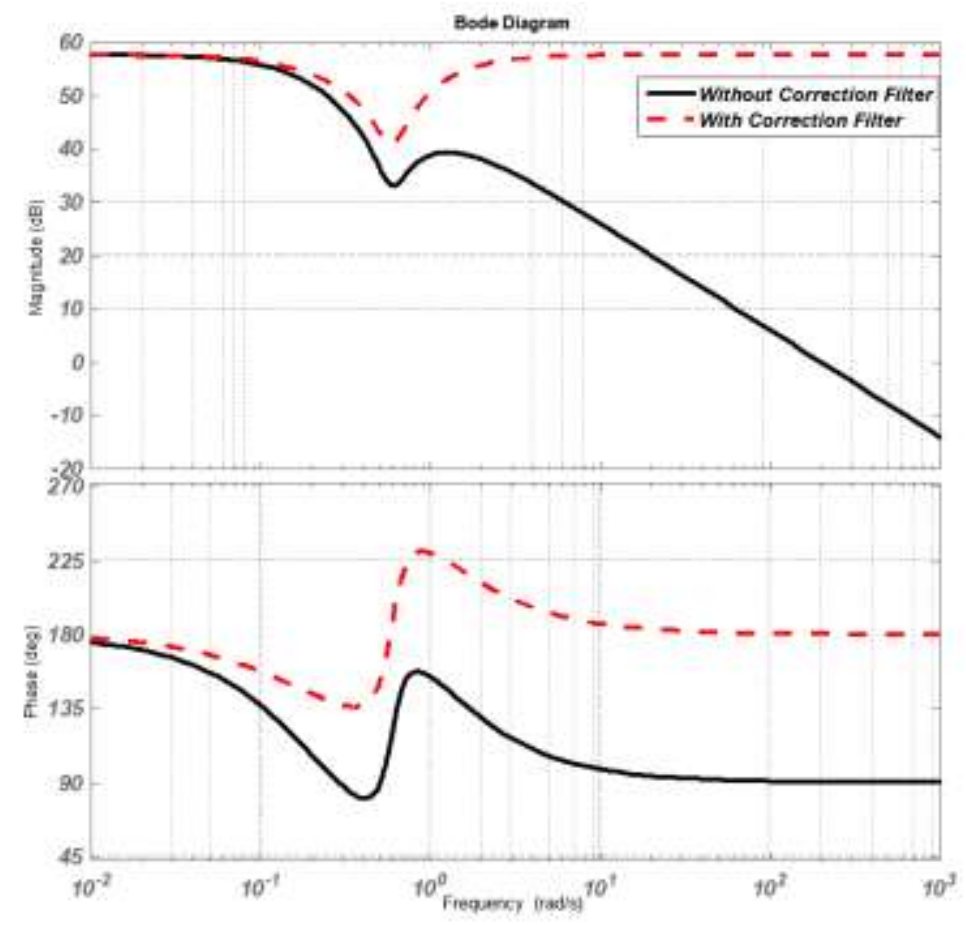

Figure 8. Frequency Response of the Main Branch (1), a) Decoupling Process, b) Decoupling With Correction Filter Process

$$
U_{P D_{2}}=K_{2} \frac{\left(S T_{7}+1\right)\left(1+S T_{8}\right)}{\left(S^{2} T_{9}^{2}+S T_{10}+1\right)}
$$

The high frequency approximations are defined by (11 and 12).

$$
\begin{aligned}
& U_{P D_{1}}(\infty)=K_{1} \frac{\left(T_{1}^{2} T_{3}\right)}{T_{4}{ }^{3}} \\
& U_{P D 2}(\infty)=K_{2} \frac{\left(T_{7} T_{8}\right)}{T_{9}{ }^{2}}
\end{aligned}
$$

Based on (11 and 12) in high frequency domain the controller behavior is close to the proportional control algorithm and have maximum bandwidth. The time constant of the filters are defined by (13 and 14).

$$
\begin{gathered}
T_{3}=\frac{T_{4}{ }^{3}}{T_{1}{ }^{2}} \\
T_{8}=\frac{T_{9}{ }^{2}}{T_{7}}
\end{gathered}
$$




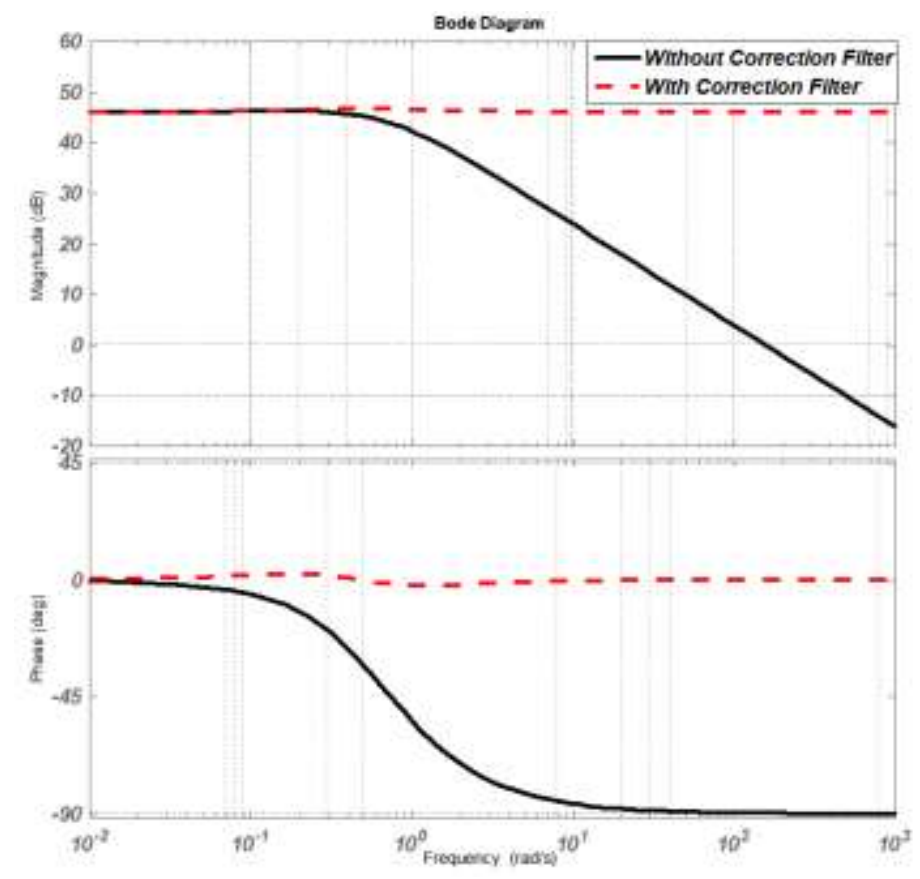

Figure 9. Frequency Response of the Main Branch (2), a) Decoupling Process, b) Decoupling With Correction Filter Process

Regarding to (13 and 14) in Figures 8 and 9, the filters increase the gain to have a stable response in high frequencies. Based on (7), (13) and (14) this method identical the low and high frequency gains in distillation column. The next step is focused on the design I-controller and applied to filter PD type. The main idea of this theory is defined as the following equations:

$$
\begin{aligned}
& U_{I_{1}}(S)=\frac{1}{1+S T_{11}}, T_{11}=\frac{1}{K_{1} K_{I_{1}}} \\
& U_{I_{2}}(S)=\frac{1}{1+S T_{12}}, T_{12}=\frac{1}{K_{2} K_{I_{2}}}
\end{aligned}
$$

where $K_{I_{1}}$ and $K_{I_{2}}$ are the integral action factor for first controller and second controller, respectively. The next part is focused on the design PID like fuzzy scheduling to best tuning the coefficients and rise-time for filters. The main idea of fuzzy logic theory is defined as follows:

$$
\left[Y_{F u z z y}\right]=\sum_{1}^{X} \gamma^{T} \lambda(x)
$$

where $\gamma^{T}$ and $\lambda(x)$ are the fuzzy coefficient factor and the fuzzy main function, respectively, that is defined by the following equation.

$$
\lambda(x)=\frac{\sum_{i} \mu(x) \cdot x}{\sum_{i} \mu(x)}
$$

Here, $\mu(x)$ is the degree of membership in the fuzzy logic design theory. 


$$
\left[Y_{F u z z y}\right]_{P I D}=\sum_{1}^{X} \gamma^{T} \lambda(e, \dot{e})+\sum_{1}^{X} \gamma^{T} \lambda\left(e, \sum e\right)
$$

To have the best convergence, the coefficients defined by the following formulation.

$$
K_{i_{1}}=\frac{K_{1}}{T_{a_{1}}}, K_{i_{2}}=\frac{K_{2}}{T_{a_{2}}}
$$

where $T_{a_{1}}$ and $T_{a_{2}}$ are rise time for PD filter and I-controller for first and second controller, respectively. To design fuzzy scheduling, three different factors are defined based on the below definitions:

$$
\begin{gathered}
K_{i_{F_{1}}}=\frac{K_{1}^{2}}{\beta_{1} \cdot K_{i_{1}}}, \beta_{1}=\frac{T_{a_{s_{1}}}}{T_{a_{1}}} \\
K_{i_{F_{2}}}=\frac{K_{2}^{2}}{\beta_{2} \cdot K_{i_{2}}}, \beta_{2}=\frac{T_{a_{s_{2}}}}{T_{a_{2}}}
\end{gathered}
$$

The normalization functions are defined by (22 and 23)

$$
\begin{aligned}
& K_{1}^{\prime}=\frac{K_{1}-K_{1(\min )}}{K_{1(\max )}-K_{1(\min )}} \in[0,1] \\
& K_{i_{1}}^{\prime}=\frac{K_{i_{1}}-K_{i_{1}(\min )}}{K_{i_{1}(\max )}-K_{i_{1}(\min )}} \in[0,1] \\
& 2 \leq \beta_{1} \leq 5 \\
& K_{2}^{\prime}=\frac{K_{2}-K_{2(\min )}}{K_{2(\max )}-K_{2(\min )}} \in[0,1] \\
& K_{i_{2}}^{\prime}=\frac{K_{i_{2}}-K_{i_{2}(\min )}}{K_{i_{2}(\max )}-K_{i_{2}(\min )}} \in[0,1] \\
& 2 \leq \beta_{2} \leq 5
\end{aligned}
$$

To find above coefficients PID like fuzzy logic have been designed based on (24) and (25).

$$
\begin{aligned}
& K_{1}^{\prime}=\left(\sum_{1}^{X} \gamma^{T} \lambda_{K_{1}}(x) \in[0,1]\right. \\
& K_{i_{1}}^{\prime}=\left(\sum_{1}^{X} \gamma^{T} \lambda_{K_{i_{1}}}(x) \in[0,1]\right. \\
& \beta_{1}=\left(\sum_{1}^{X} \gamma^{T} \lambda_{\beta_{1}}(x)\right. \\
& K_{2}^{\prime}=\left(\sum_{1}^{X} \gamma^{T} \lambda_{K_{2}}(x) \in[0,1]\right. \\
& K_{i_{2}}^{\prime}=\left(\sum_{1}^{X} \gamma^{T} \lambda_{K_{i_{2}}}(x) \in[0,1]\right. \\
& \beta_{2}=\left(\sum_{1}^{X} \gamma^{T} \lambda_{\beta_{2}}(x)\right.
\end{aligned}
$$

Figures 9 and 10 show the power of disturbance rejection in multivariable decoupling filter fuzzy PID scheduling for distillation columns.

According to Figures 9 and 10, proposed method is stable and robust for uncertain condition in distillation column. This control algorithm has a good disturbance rejection with fast response in flow and concentration. Without control system the deviation in temperature is more than 200 but with controller the deviation in temperature reduce to 1 . To checking the power of stability in distillation column, step response and bode diagram of distillation column are shown in Figures11 and 12. According to Figure11, multi 
variable filter fuzzy PID scheduling is a robust and stable decouple controller for our nonlinear system. The output temperature variation is limited when we applied this control methodology. Based on Figure 12 we can see that, the resonance frequency for two branches are the same and close to about $10 \mathrm{~Hz}$ and based on this graph, this system is very robust to parameter variation.

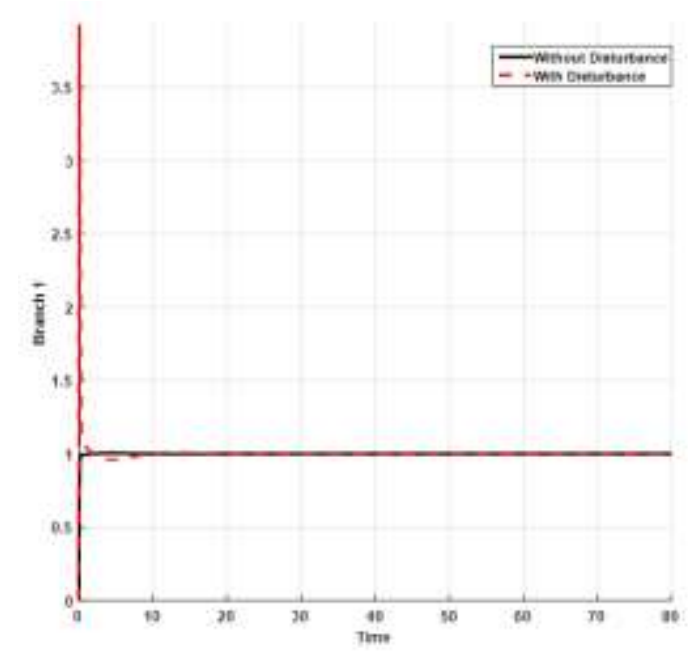

Figure 9. Disturbance Rejection Response of the Main Branch (1), a) without Disturbance, b) with Disturbance

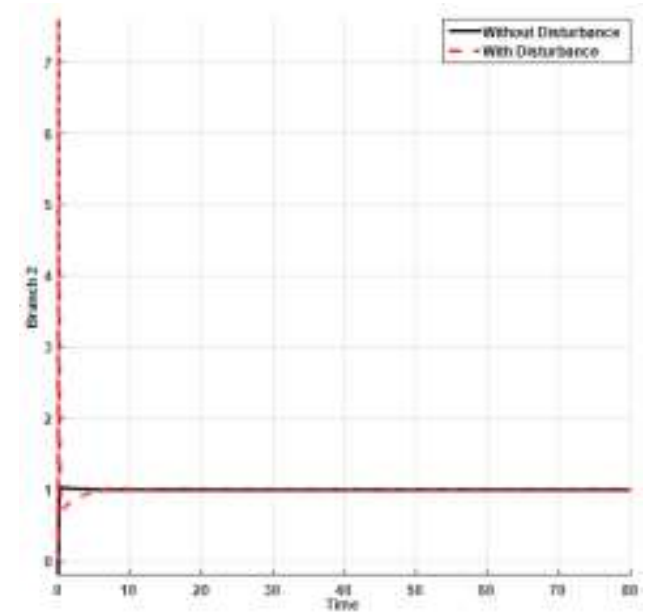

Figure 10. Disturbance Rejection Response of the Main Branch (2), a) without Disturbance, b) with Disturbance 


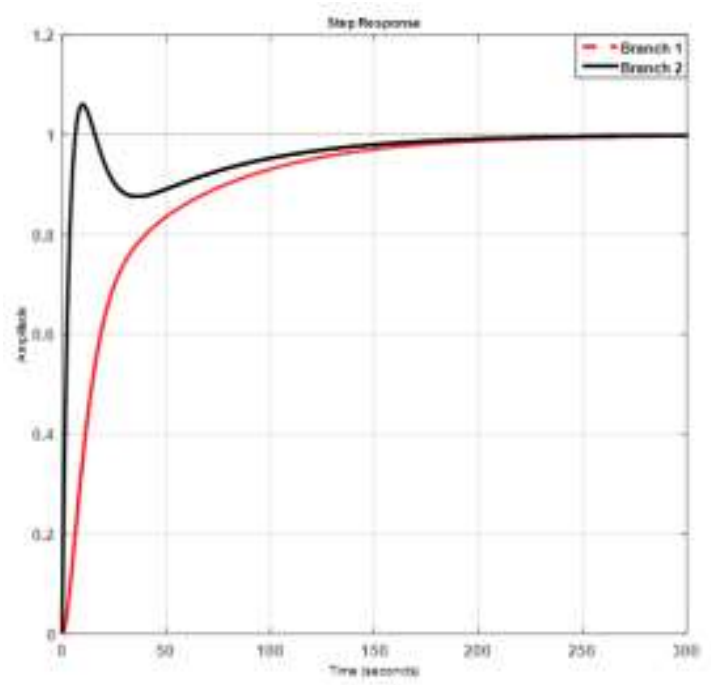

Figure 11. Step Response of the Main Branch (1) and (2) for Distillation Column

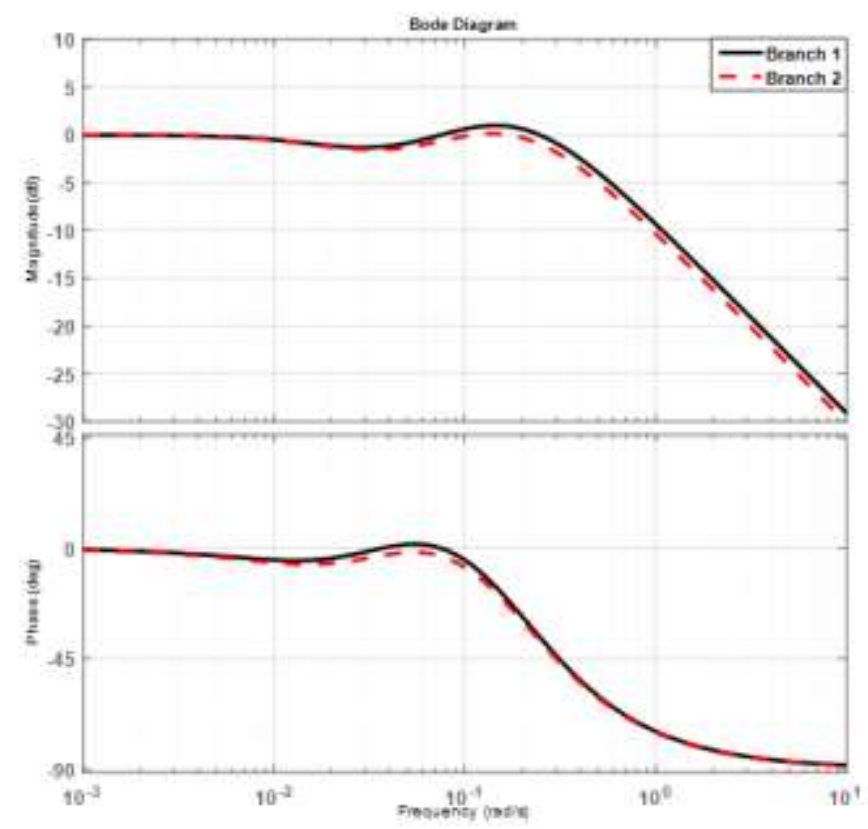

Figure 12. Frequency Response of the Main Branch (1) and (2) for Distillation Column

\section{Conclusion}

This study proposes a method to solve two significant challenges. The first challenge is the coupling effect in a distillation column. To address this challenge, the multivariable decoupling method was recommended. Although this method has positive attributes, dynamic cross-over between two parts of the circuit remain challenge. A low pass filter is presented to solve this challenge. This method eliminates the dynamic cross-over and improve the performance of multivariable decoupling method. The second challenge is improved the temperature controllability and observability in distillation column. A fuzzy PID scheduling controller is applied to multivariable filter decoupling method to solve this challenge. This approach reduces the variation in output temperature and improves the rise-time in the certain and uncertain condition. The frequency and phase response 
have been tested and approved the power of stability and robustness in modified multivariable filtered fuzzy PID scheduling control distillation column.

\section{Acknowledgement}

This work was supported by the Korea Institute of Energy Technology Evaluation and Planning (KETEP) and the Ministry of Trade, Industry \& Energy (MOTIE) of the Republic of Korea (No. 20162220100050, No. 20161120100350, and No. 20172510102130). It was also funded in part by the Leading Human Resource Training Program of Regional Neo Industry through the National Research Foundation of Korea (NRF) funded by the Ministry of Science, ICT and future Planning (NRF2016H1D5A1910564), and in part by the Basic Science Research Program through the National Research Foundation of Korea (NRF) funded by the Ministry of Education (2016R1D1A3B03931927).

\section{References}

[1] C. S. Bîldea, "Cyclic distillation technology-a mini-review", Journal of Chemical Technology and Biotechnology, vol. 91, no. 5, (2016), pp. 1215-1223.

[2] R.-J.Yang, "A comprehensive review of micro-distillation methods", Chemical Engineering Journal, vol. 313, (2017), pp. 1509-1520.

[3] P. Acharya, G. Dumpa and T. Kumar Dan, "Modelling and control of distillation column", Computation of Power, Energy Information and Commuincation (ICCPEIC), 2016 International Conference on. IEEE, (2016).

[4] B. E. Park, S. W. Sung and I.-B. Lee, "Design of centralized PID controllers for TITO processes", Advanced Control of Industrial Processes (AdCONIP), 2017 6th International Symposium on. IEEE, (2017).

[5] J. Drgoňa, "Fuzzy control of a laboratory binary distillation column", Process Control (PC), 201721 st International Conference on. IEEE, (2017).

[6] S. Banerjee and A. K. Jana, "Internally heat integrated batch distillation: Vapor recompression and nonlinear control", Separation and Purification Technology, vol. 189, (2017), pp. 267-278.

[7] S. Banerjee and A. K. Jana, "Dynamic vapor recompression in a reactive batch rectifier: Analysis and nonlinear control”, Energy, vol. 115, (2016), pp. 60-66.

[8] Y. Li, S. Sui and S. Tong, "Adaptive fuzzy control design for stochastic nonlinear switched systems with arbitrary switchings and unmodeled dynamics", IEEE transactions on cybernetics, vol. 47, no. 2, (2017), pp. 403-414.

[9] S. Yin, P. Shi and H. Yang, "Adaptive fuzzy control of strict-feedback nonlinear time-delay systems with unmodeled dynamics", IEEE transactions on cybernetics, vol. 46, no. 8, (2016), pp. 1926-1938.

[10] Q. Zhou, "Adaptive fuzzy control for nonstrict-feedback systems with input saturation and output constraint”, IEEE Transactions on Systems, Man, and Cybernetics: Systems, vol. 47, no. 1, (2017), pp. $1-12$.

[11] J. Shen, "Speed Control of Single-axis Rotation INS by Tracking Differentiator Based Fuzzy PID", IEEE Transactions on Aerospace and Electronic Systems, (2017).

[12] S. Ciannella, "Using hot-vapor bypass for pressure control in distillation columns", Chinese Journal of Chemical Engineering, (2017).

[13] Y. Yuan, "Asymmetrical temperature control of a BTX dividing-wall distillation column", Chemical Engineering Research and Design, vol. 123, (2017), pp. 84-98.

[14] E. Ebrahimzadeh and L. L. Baxter, "Plant-wide control of coupled distillation columns with partial condensers", Applied Thermal Engineering, vol. 102, (2016), pp. 785-799. 


\section{Authors}

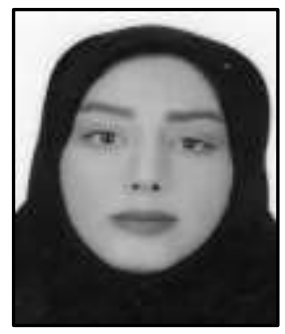

Niloofar Mirzavand, she is a researcher at the IRANSSP research and development center. Her research interests include chemical engineering process, industrial control technique, artificial intelligence, and system modeling.

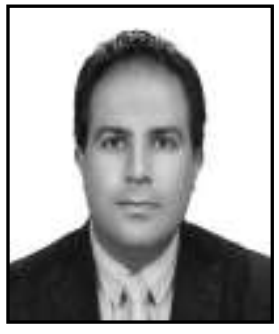

Farzin Piltan, he is a research associate at the department of Electrical and Computer Engineering, at the University of Ulsan, Ulsan, South Korea. He is also the senior researcher and president of the IRANSSP research and development center, Shiraz, Iran. He has published more than 170 peer review research articles and nine books. He is also an editorial board member of four academic journals. His research interests include fault diagnosis, nonlinear control, system modeling, and embedded systems.

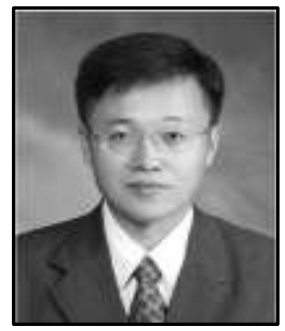

Jong-Myon Kim, (M'05) he received his B.S. degree in electrical engineering from Myongi University, Yongin, South Korea in 1995, M.S. degree in electrical and computer engineering from the University of Florida, Gainesville, FL, USA in 2000, and Ph.D. degree in electrical and computer engineering from the Georgia Institute of Technology, Atlanta, GA, USA in 2005. He is currently a professor with the Department of IT Convergence and also Vice President of the Foundation for Industry Cooperation at the University of Ulsan, Ulsan, South Korea. His research interests include multimedia-specific processor architecture, fault diagnosis and condition monitoring, parallel processing, and embedded systems. Dr. Kim is a member of the IEEE Industrial Electronics Society. 
International Journal of Control and Automation

Vol. 11, No. 1 (2018) 